\title{
Statistical Estimation of Residual Strength and Reliability of Corroded Pipeline Elbow Part Based on a Direct FE-Simulations
}

\author{
O. Larin ${ }^{1}$, K. Potopalska ${ }^{1 *}$, R. Mygushchenko1 \\ ${ }^{1}$ National Technical University "Kharkiv Polytechnic Institute" \\ e-mail: alexeya.larin@gmail.com \\ e-mail: ks.potopalskaya@gmail.com \\ e-mail:mrp1@ukr.net \\ *corresponding author
}

\begin{abstract}
In this paper, the assessment of the effect of a corrosion defect, which is developing over time on the working capacity of the pipeline elbow, has been carried out. Corrosion damage models explicitly as volumetric defect on the out surface of the pipeline elbow. The zones on the damaged section of the pipeline on which the maximum stresses are localized has been defined using the finite element analysis. The estimation of the characteristics of the deformed state of a design with defect is analysed with the framework of computer simulations. On the basis of the obtained results, the probabilistic characteristics of the stresses and plastic strains have been approximated as a function of internal pressure and corrosion defect depth, such as mean value, coefficients of variation and asymmetry. Probability densities function of plasticity appearance have been found for different defect size in operation at typical loading levels.
\end{abstract}

Keywords: Corrosion damage, pipeline, reliability, volumetric surface defect, plastic strains

\section{Introduction}

Pipelines are one of the most popular way for transporting oil and gases. During long-term operation, damage to the pipeline can occur because of the accumulation of fatigue and the occurrence of corrosion on the surface of the structural elements. This may lead to cracks and depressurization of construction. Timely diagnosis of such damage can prevent the emergencies, environmental disasters, pollution and economic losses. Providing a maintenance and repair of such a system in required time is a very important practical task.

Appearance of corrosion defects, the features of their growth and the effect on residual durability structural elements are of interest for engineers (Larin \& Vodka, 2015). The scientists focus on analyzing and predicting the initiation of corrosion, as well as modeling its growth. The study of the causes of the origin of corrosion are in the competence of physical chemistry and materials science. In this case, the determination of the technological means for increasing the stability of the material to oxidation or other chemical reactions are considered. A lot of attention is also paid for a process of the growth of corrosion defect (da Costa-Mattos, Reis, Sampaio, \& Perrut, 2009; Larin, Barkanov, \& Vodka, 2016; Netto, Ferraz, \& Botto, 2007). Here are experimentally analyzed the rate of corrosion growth, as well as the influence of external factors on it (Chen et al., 2015). On the other hand, there are some mathematical modeling approaches of the corrosion 
growth process that can be used to predict reliability and to assess the residual strength of structures that are already damaged. In recent studies, the residual strength of a structure is often estimated by explicit modeling of corrosion defect (Caleyo, Velázquez, Valor, \& Hallen, 2009; De Leon \& Macías, 2005; Khalaj Khalajestani \& Bahaari, 2014; Li, Yu, Zeng, Li, \& Liang, 2009; Ma, Shuai, Liu, \& Xu, 2013; Silva, Guerreiro, \& Loula, 2007; A. Valor, Caleyo, Alfonso, Rivas, \& Hallen, 2007; Vodka, 2015), which is usually carried out in CAE software using the finite element method (FEM). This approach allows investigating the changes in the deformed state of a pipeline with defects of various shapes and in different locations. The studies are focused on the assessment of the residual strength of pipelines with corrosion defects in its straight part of pipe (Ahammed, 1997; da Costa-Mattos et al., 2009; Mirchev, Larin, \& Potopalska, 2016). There are works which analyze the residual strength of the elbow of the pipeline, and it has been shown that a corrosion on the curvilinear part of the pipeline results a higher concentration of stress (Cunha, Benjamin, Silva, Guerreiro, \& Drach, 2014; Khalaj Khalajestani \& Bahaari, 2014). But in these studies defects were considered with fixed dimensions, but corrosion is a process that should be considered depending on the operating time. Thus, it is important to carry out a research for estimation of the strength of curved part of the pipeline with simulation of the process of possible corrosion damage growth based on three-dimensional FE models. The appearance and development of the defect is affected by many external factors, such as the environment, the variation of the operating load, and so on. Therefore, the development of surface corrosion defects should be considered in a probabilistic statement.

The aim of this work is to assess the residual strength of the damaged elbow of the pipeline on the basis of statistical estimation of the concentration of the stresses and plastic strains of the design with a corrosion defect, which is given with random dimensions, depending on the time of its occurrence. A parametric FE model is developed. It has a complex 3D curvilinear geometry and a 3D volumetric defect. Assessment of its impact on residual structural strength is based on statistical Monte Carlo modeling, taking into account the operational load and the statistical nature of the corrosion damage dimensions.

The solution of this problem can be divided as it is shown in Fig. 1.

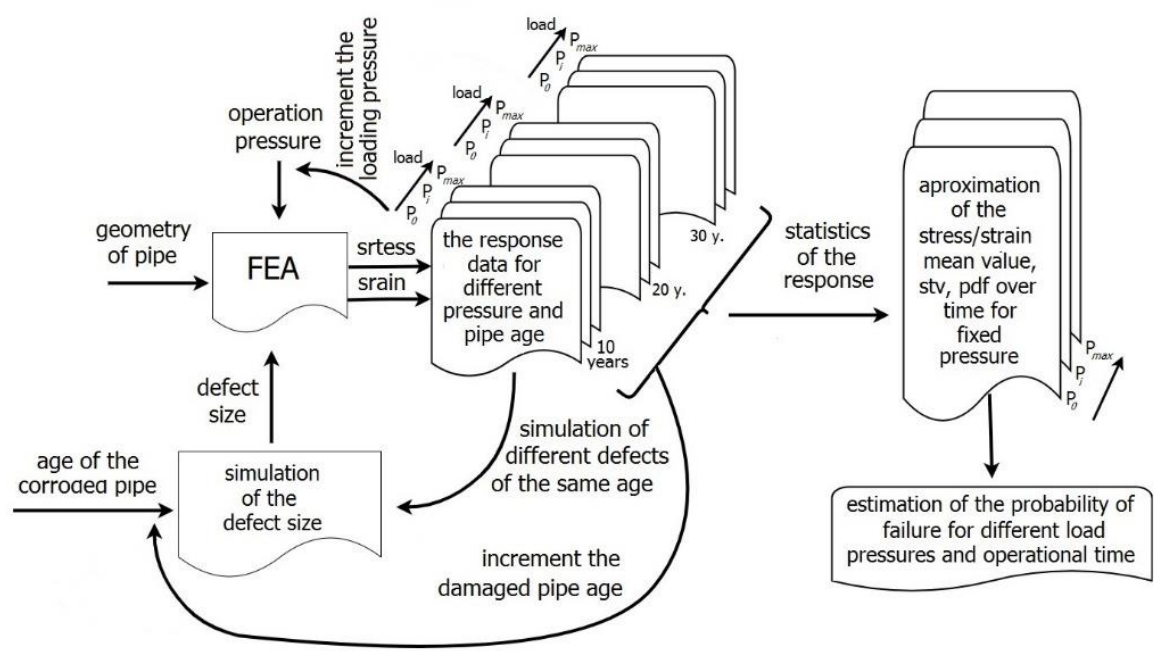

Fig. 1. Scheme for solving the problem 
This scheme shows stages of investigation for estimation of a probability of failure of damaged pipeline elbow.

1. On the first stage the parametric model of pipeline elbow with volumetric defect is created which allows to automatically make a FE analysis of deformed state of the pipe with defect of different size. The dimensions of defect (depth, length and weight) is simulated from known statistics possible damage size of such an age and environment. Nominal internal pressure is set a load.

2. On the second stage, using the developed model a number of varied solutions in ANSYS CAE software for different load level has been made. For that purpose, the static simulation was made for model with corresponding dimensions' form stage 1 and with linearly changed load.

3. The step 2 repeated at least 150 times with the generation of different defects dimension, which probably can occur in operation of pipeline of analysed age.

4. On the next stage the defect age is increased and new sets of pipes with defects with different dimensions are generated and their deformed state is analysed. For the steps 3 and 4 inserted loops on each incremented defect age are repeated.

5. The accumulated on the steps 2-4 gives us the statistics of stress concentration in defected pipeline with corrosion damage has some known age of growth.

6.Further, to process statistics an approximation of the way stress values in defect and plastic strain values as a functions of defect sizes and internal pressures. On this stage using obtained probabilistic parameters, the probability density function of deformed state parameter is determined.

7. Finally, on the basis of the obtained numerical dependencies, an estimation of the probability of failure of a damaged pipeline part can be made as a function of time and internal pressure.

\section{Fundamental relations}

\subsection{Corrosion defect modeling}

The specific dimensions of the defect are determined from it pre-analysis to study the residual durability of the pipeline at a certain time of its operation. From the analysis of data presented in literature, it can be summarized that linear (Li et al., 2009) or nonlinear (Bazán \& Beck, 2013; Alma Valor, Caleyo, Hallen, \& Velázquez, 2013) models are usually used to estimate corrosion kinetics defects growth.

In this paper, it is proposed to take into account the growth of the defect over the time using a power function, which binds the operating time and the defect sizes (Caleyo et al., 2009):

$$
d_{j}(t)=k\left(t-t_{0}\right)^{\alpha}
$$

where $t_{0}$ time of occurrence of corrosion damage, $\alpha$ and $k$ are parameters of defect kinetics. From practice of pipelines exploitation, it is known that typically within 5 years on the surface of the pipeline corrosion damage appears $\left(t_{0}=5\right.$ years). The process of corrosion growth is random so far, the parameters of the kinetics equation: $\alpha$ and $\mathrm{k}$ should be considered as random variables. It is also known (Bazán \& Beck, 2013; Teixeira, Guedes Soares, Netto, \& Estefen, 2008) some statistics about the possible values of these parameters and their variations. Moreover, for different directions of corrosion defect growth (such as, depth and width along the surface). 
The parameter $k$ can change substantially, even in identical external factors. The parameter $\alpha$ is considered to have a constant deterministic value (Bazán \& Beck, 2013), but there is a work that analyzes it from a statistical point of view (Teixeira et al., 2008). However, its variation is not significant; therefore, in this paper, it is suggested that the parameter $\alpha$ it set to be fixed and equal to 0.73 (Bazán \& Beck, 2013; Cunha et al., 2014).

From the literature it is also known that the statistics of the values of the dimensions of the defect with sufficient accuracy can be considered to the lognormal distribution (Teixeira et al., 2008)

$$
f\left(d_{j}, t\right)=\frac{1}{d_{j} S_{j}(t) \sqrt{2 \pi}} \exp \left[\frac{\left(-\ln d_{j}-\mu_{j}(t)\right)^{2}}{S_{j}^{2}(t)}\right]
$$

where $S_{j}(t)$ and $\mu_{j}(t)$ are the parameters of the law depending on the size of the defect in the current operation time and are determined by the equation (1). The coefficient of variation and mathematical expectation of the defect dimensions one can find in the following manner:

$$
\begin{gathered}
\mu_{j}(t)=\ln \left(\frac{m_{j}(t)}{\left.\sqrt{\frac{v_{j}}{m_{j}^{2}(t)}}\right)}, \quad S_{j}^{2}(t)=\ln \left(1+\frac{v_{j}}{m_{j}^{2}(t)}\right),\right. \\
m_{j}(t)=\left\langle k_{j}\left(t-t_{0}\right)^{\alpha}\right\rangle=\left\langle k_{j}\right\rangle\left(t-t_{0}\right)^{\alpha} \\
\operatorname{Var}_{j}(t)=\left\langle\left(k_{j}\left(t-t_{0}\right)^{\alpha}\right)^{2}\right\rangle_{j} \operatorname{Var}_{k}\left(t-t_{0}\right)^{2 \alpha} \\
v_{j}=\frac{\sqrt{\operatorname{Var}_{d}(t)}}{m_{j}(t)}=\frac{\sqrt{\operatorname{Var}_{k}(t)}}{\left\langle k_{j}\right\rangle}=\text { const }
\end{gathered}
$$

where $\langle\ldots\rangle$-the operator of averaging, $m_{j}(t)$ - mathematical expectation, Var ()$-$ the operator of variance, $v_{j}$-coefficient of variation of $d_{j}$ defect dimension. Figure 2 schematically shows the development over time of the average depth of corrosion damage $m_{3}(t)$ and the possible spread of its values. 


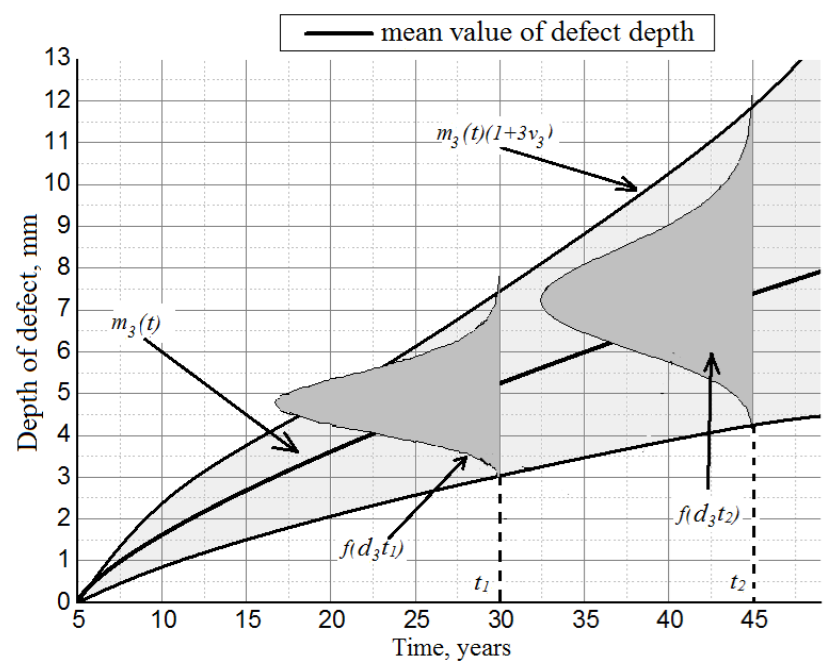

Fig. 2. The possible change in depth of defect over time of pipe operation.

Therefore, it is proposed to develop FE models with a defect of a certain level of defect growth state.

\subsection{FE-modelling}

A fragment of the main pipeline elbow is modelled with geometry, material properties and loads, which correspond to ASME B31G. The model has been composed of toroidal and cylindrical parts. The following values of the structure dimensions were used: outside radius of the pipeline $D=508 \mathrm{~mm}$; wall thickness $h=12.5 \mathrm{~mm}$; length of the studied pipeline part $L=1.5 \mathrm{~m}$. Those correspond to the X42 pipe grade. The length of the straight part of the pipe section is chosen posterior after a number of preliminary tests when edge effects in the model do not influence the deformed state in the investigated section of the pipe. The material is steel, the properties of which are given in Table 1.

\begin{tabular}{|c|c|c|c|c|}
\hline Parameter & Young's modulus & Poisson ratio & Yield stress & Ultimate stress \\
\hline Symbol & $E, \mathrm{GPa}$ & $v$ & $\sigma_{y}, \mathrm{MPa}$ & $\sigma_{u}, \mathrm{MPa}$ \\
\hline Value & 205 & 0.3 & 290 & 430 \\
\hline
\end{tabular}

Table 1. Properties of pipeline material

The pipeline section is fixed at the edges and loaded with internal pressure P. For such pipelines, the nominal (working) pressure is $3 \mathrm{MPa}$. It should also be noted that at moments of start and stop internal pressure essentially overestimates the nominal value. The level of maximum load depends on the location of the pipeline and, according to ASME B31.8 (2003), may vary from 6 $\mathrm{MPa}$ to $12.8 \mathrm{MPa}$ (Table 2), where LC1-LC4 is the typical possible location of the pipes elbow.

\begin{tabular}{|c|c|c|c|c|c|}
\hline Location area & Typical value & LC1 & LC2 & LC3 & LC4 \\
\hline Maximum pressure, MPa & 6 & 12.8 & 10.6 & 8.6 & 7.1 \\
\hline
\end{tabular}

Table 2. Change of internal pressure 
The volumetric superficial defect is modeled in the center of the outer surface of the elbow of the pipeline (Fig. 3). A defect is chosen for the study in the form of a parallelepiped with rounded borders. Since the defect is located on the bend of the pipe, the sketch was designed in the local toroidal coordinate system, which depends on the major radius of the elbow of the pipe $R$. Geometric parameters such as length $\left(d_{1}\right)$, width $\left(d_{2}\right)$ and depth $\left(d_{3}=h-d\right)$ are given parametrically. This allows to automatically rebuild the model for different size of defect.

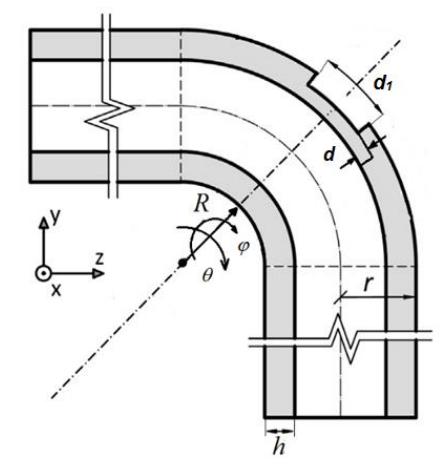

Fig. 3. Sketch of computational model.

FEM has used to determine the stress strain state of the curvilinear part of the pipeline. In the calculations, a half of the model was used, which takes into account the pipeline's symmetry.

The size of the elements gradually decreases near the damaged zone. It is given four elements over the thickness. On undamaged sections of the pipeline, a mapped hexagonal mesh was constructed, in the defect zone - tetrahedral. Iso-parametric finite elements with 8 nodes and 3 degrees of freedom at the node was used. The mesh of the whole model and a detailed defected zone are depicted in Fig.4.

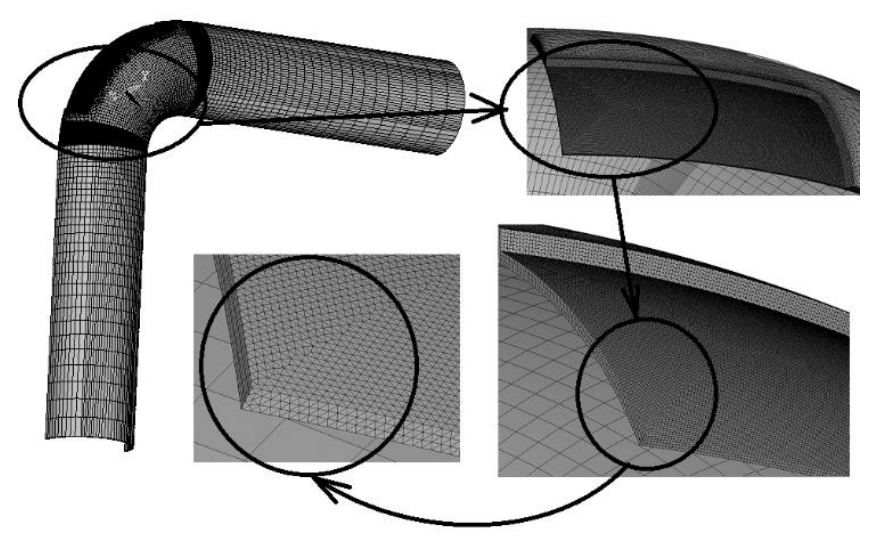

Fig. 4. The mesh of the quarter of pipeline elbow.

\section{Results and discussion}

Prior to statistical simulations, a series of calculations of the pipeline's elbow defects with mean dimensions has been performed in accordance with the geometric parameters corresponding to 
different time on operation of the damaged pipeline (from 10 to 45 years). It is revealed that plastic strains firstly appear in the longitudinal direction of damage. It should also be claimed that when the structure is used up to 23 years and has a defect with fixed dimensions, plastic strains begin to appear in the opposite direction relative to the defect, that is, in the place of highest curvature. Moreover, these strains appear for large values of internal pressure, which do not meet the conditions of operation. Thus, we can conclude that the surface defect with average overall dimensions during the first 23 years does not significantly affect the pipe strength. In fig. 5 it is shown at which load equivalent stresses reach the yielding point and overall strength of design with the mean defect size.

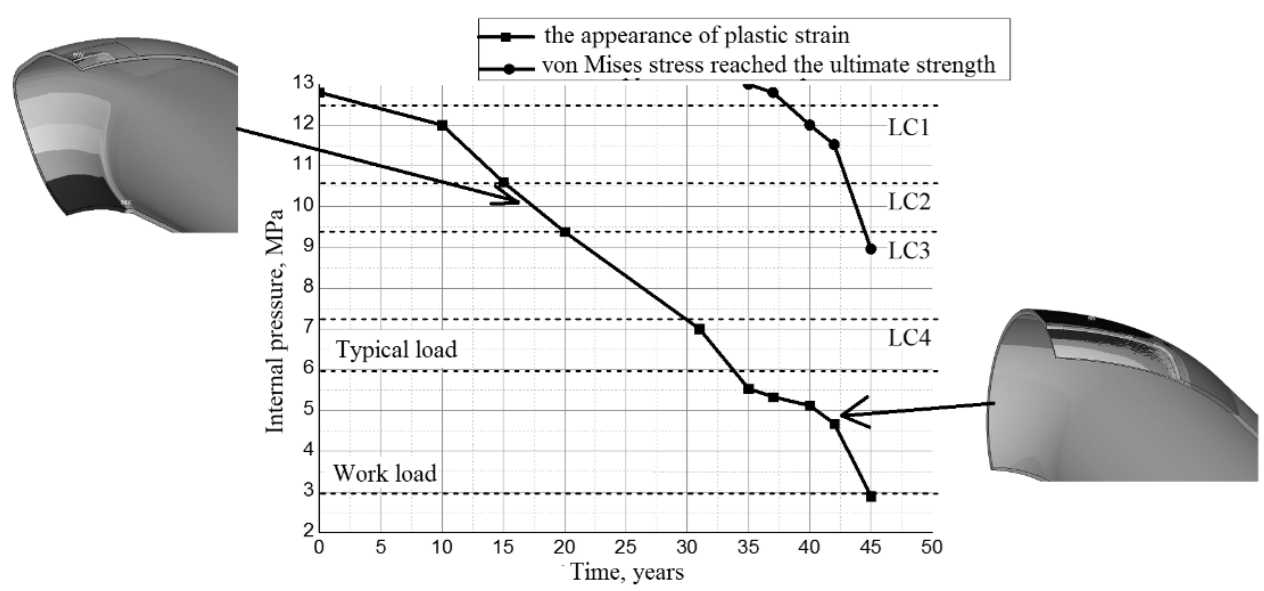

Fig. 5. The level of the internal pressure, which leads to the appearance of plastic strain in the design and the strength limit with the average dimensions of the box defect.

On Figure 5, the line with rectangular markers reflects a change in the value of internal pressure, in which plastic strain occurs in the design during the corresponding period of operation. The straight lines indicate the maximum external load, depending on the typical location of the pipeline.

It was found that in the period after 35 years of operation in the pipeline, plastic strains may occur at the typical maximum load (Fig. 5 line with rectangular markers). In the period of operation from 37 years in the pipeline with a corresponding defect under the action of the maximum possible load, there are tensions that reach the ultimate stress (Fig. 4 line with round markers).

Therefore, further investigations of the damaged pipeline were carried out for a period of operation from 35 to 45 years. For each time point (from 35 to 45 years), approximately 200 calculations with random size of the defect were performed which are subject to the lognormal distribution and have corresponding known probabilistic characteristics (3-4).

For the analysis of the results, 5 points (K, L, H, G, F in Fig. 7) were selected on the design in the defect zone located in the typical locations of appearing of plastic strain that were detected in previous studies.

After scheme of statistical simulations, the probabilistic characteristics of the design of the deformed state are obtained at different load levels and at different operating periods. The obtained data on the equivalent stress according to the Mises criterion, have been statistically processed. Histograms has been formed (fig. 6) for different load levels and determined the mean value, variance and asymmetry coefficients. 


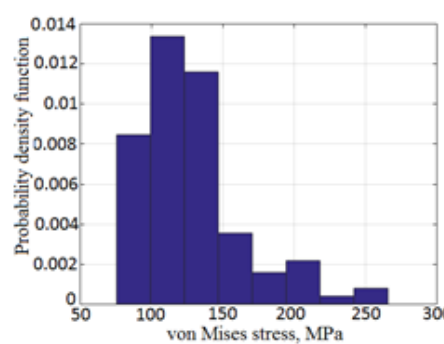

a) Working load ( $3 \mathrm{MPa})$

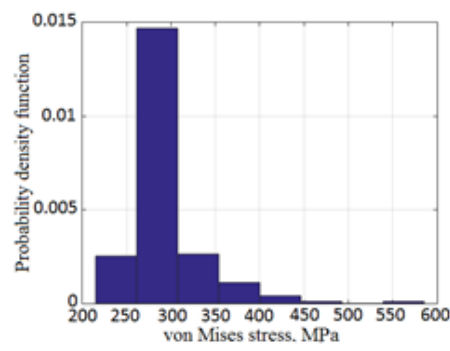

d) $\mathrm{LC} 3(8.6 \mathrm{MPa})$

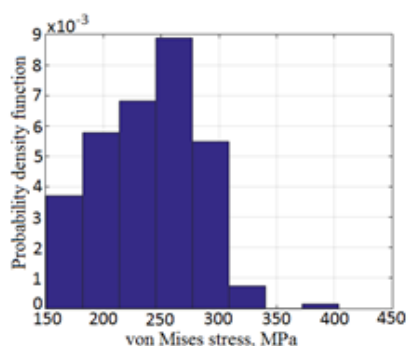

b) Start/stop (6 MPa)

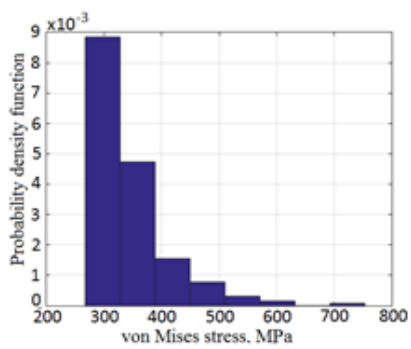

e) LC2 (10.6 MPa)

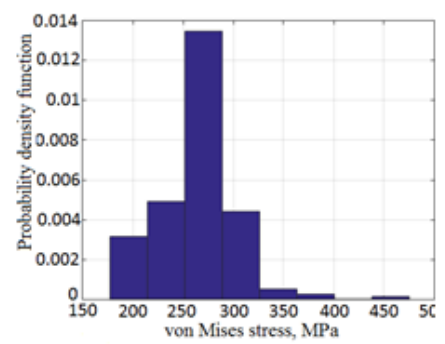

c) LC4 (7.1 MPa)

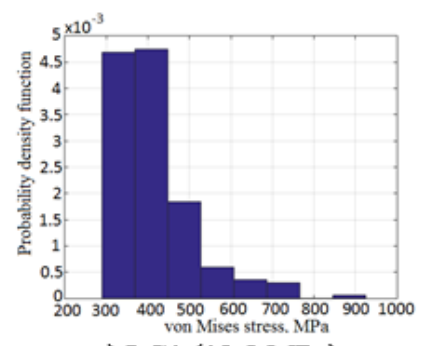

g) LC1 (12.8 MPa)

Fig. 6. Histograms of the maximum equivalent stresses in a damaged pipeline after 45 years operation at different load levels

Fig. 7 shows the dependence of the mean value of stresses on internal pressure. From the obtained results it was determined that during operation of the pipeline at the maximum load from 35 years von Mises stress arising to yield stress. The function of mean value was determined by a polynomial approximation.

$$
m_{\sigma_{e q}}(p, t)=K_{m 1}(t) p^{3}+K_{m 2}(t) p^{2}+K_{m 3}(t) p+K_{m 4}(t) \text { for } 2 M P a \leq p \leq 13 M P a
$$

The graphs (fig. 7) are shown that the nature of the stress changing is similar in considered cases. But, it should be mentioned that, increasing of operation time makes a scatter of equivalent stress value in the corresponding points broader. In longitudinal direction of defect (points K, L, M) the equivalent stress increases faster than in circumferential direction (points $G, F$ ). In point $M$, the highest mean value of von Mises stresses has been observed, so in further calculations this point has been considered. The parameters of approximation $K_{m i}$ are shown on table 3 . 


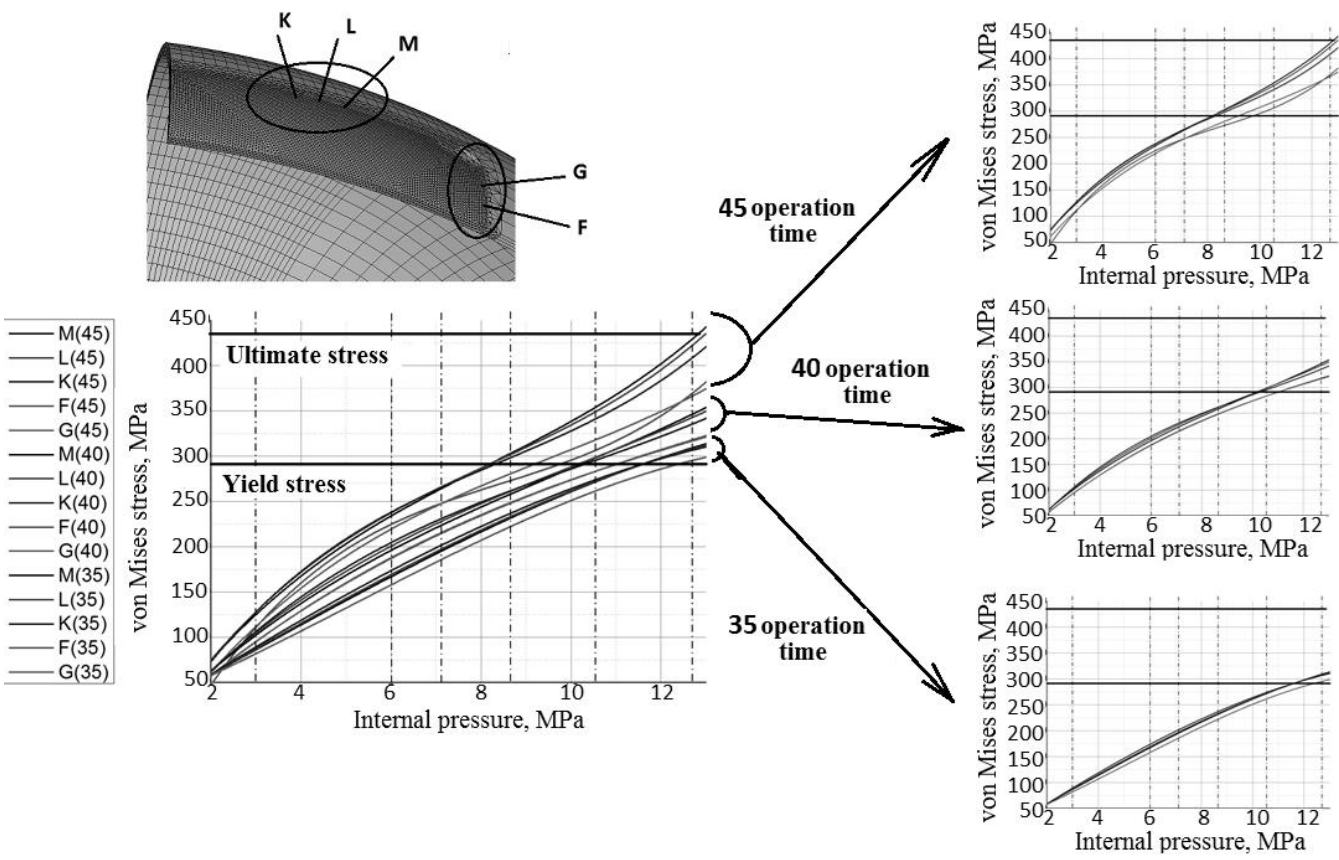

Fig. 7. Mean value of von Mises stresses in the defect at different periods of operation.

\begin{tabular}{|c|c|c|c|c|}
\hline Operation time & $K_{m 1}$ & $K_{m 2}$ & $K_{m 3}$ & $K_{m 4}$ \\
\hline 35 & $-0,04274$ & 0,056397 & 31,84927 & $-4,55498$ \\
\hline 40 & 0,165061 & $-4,67386$ & 65,63709 & $-50,5557$ \\
\hline 45 & 0,308114 & $-7,58975$ & 85,26311 & $-67,625$ \\
\hline
\end{tabular}

Table 3. Parameters of approximation of mean value for point $\mathrm{M}$

Fig. 8 presents the coefficients of variation and asymmetry of histograms of stresses in the defect. From the obtained results it can be concluded that at the pressure from $6 \mathrm{MPa}$ to $9 \mathrm{MPa}$ the distribution of values of equivalent stresses is narrowband, which means that in this load range the value of the equivalent stresses is close and reaches the yield stress limit.

The dependence of the asymmetry coefficient of the stress from the internal pressure (Fig. 7b) shows that the distributions are deviating to the left when the load is from $2 \mathrm{MPa}$ to $4 \mathrm{MPa}$ and from $9 \mathrm{MPa}$ to $12 \mathrm{MPa}$. And for the load in the range from 4 to $9 \mathrm{MPa}$ the distribution is almost symmetric. 


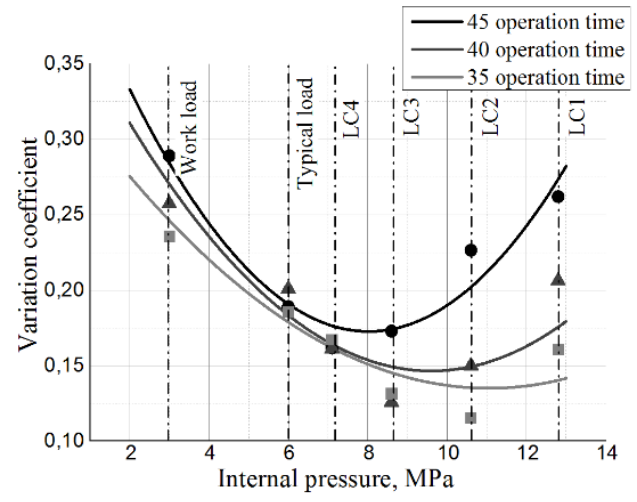

(a)

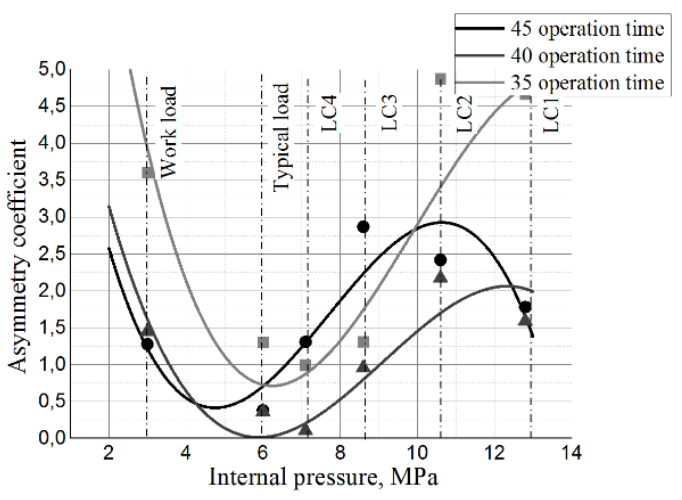

(b)

Fig. 8. Coefficients of max stresses statistics variation and asymmetry

The coefficients of variation and asymmetry were approximated by polynomials.

$$
\begin{aligned}
& v_{\sigma_{e q}}(p, t)=K_{v 1}(t) p^{2}+K_{v 2}(t) p^{1}+K_{v 3}(t) p+K_{v 4}(t) \text { for } 2 M P a \leq p \leq 13 M P a \\
& a_{\sigma_{e q}}(p, t)=K_{a 1}(t) p^{3}+K_{a 2}(t) p^{2}+K_{a 3}(t) p+K_{a 4}(t) \text { for } 2 M P a \leq p \leq 13 M P a
\end{aligned}
$$

The parameters of approximation $K_{i}$ are shown on table 4 .

\begin{tabular}{|c|c|c|c|c|}
\hline \multicolumn{5}{|c|}{ Parameters for coefficients of variation } \\
\hline Operation time & $K_{1}$ & $K_{2}$ & $K_{3}$ & $K_{4}$ \\
\hline 35 & 0,004422 & $-0,07097$ & 0,457369 & - \\
\hline 40 & 0,00344 & $-0,0613$ & 0,419505 & - \\
\hline 45 & 0,002075 & $-0,04244$ & 0,352048 & - \\
\hline \multicolumn{5}{|c|}{ Parameters for coefficients of asymmetry } \\
\hline 35 & $-0,02869$ & 0,789264 & $-6,29109$ & 16,24872 \\
\hline 40 & $-0,02035$ & 0,516743 & $-3,84843$ & 8,935208 \\
\hline 45 & $-0,02468$ & 0,569396 & $-3,73902$ & 7,97279 \\
\hline
\end{tabular}

Table 4. Parameters of approximation

Analysis of the histograms and the obtained coefficients show that, unfortunately, the statistics data are not subject directly to any typical distribution. Due to the fact that for different load levels the stress distributions are different (either it has left side asymmetry, or symmetry narrowband distribution).

Therefore, the probability density function (PDF) is proposed to approximate by orthogonal Lager series (Tihonov, 1982). Such distribution is always positively determined and based on gammadistribution (one member of the series gives), which is well suited for several considered cases. Approximation using the Lager series is as following (Tihonov, 1982):

$$
f_{1}\left(\sigma_{e q}\right)=\sum_{n=0}^{\infty} c_{n} e^{-\sigma_{e q}} \sigma_{e q}^{\alpha} L_{n}^{(\alpha)}\left(\sigma_{e q}\right)
$$


where $L_{n}^{(\alpha)}\left(\sigma_{e q}\right)$ - is a generalized Lager polynomial function, $\sigma_{e q}$ - random variable (maximum stresses in defect), $\alpha$ - arbitrary constant, $c_{n}$ - coefficient of decomposition. The generalized Lager polynomial has the following form:

$$
L_{n}^{(\alpha)}\left(\sigma_{e q}\right)=e^{\sigma_{e q}} \frac{\sigma_{e q}^{-\alpha}}{n !} \frac{d^{n}}{d \sigma_{e q}^{n}}\left(e^{-\sigma_{e q}} \sigma_{e q} n+\alpha\right)
$$

It is sufficient to be restricted to the first four members of the series:

$$
\begin{aligned}
& L_{0}^{(\alpha)}\left(\sigma_{e q}\right)=1 ; \\
& L_{1}^{(\alpha)}\left(\sigma_{e q}\right)=1+\alpha-\sigma_{e q} ; \\
& 2 L_{2}^{(\alpha)}\left(\sigma_{e q}\right)=(\alpha+1)(\alpha+2)-2 \sigma_{e q}(\alpha+2)+\sigma_{e q}{ }^{2} ; \\
& 6 L_{3}^{(\alpha)}\left(\sigma_{e q}\right)=(\alpha+1)(\alpha+2)(\alpha+3)-3 \sigma_{e q}(\alpha+2)(\alpha+3)+3 \sigma_{e q}{ }^{2}(\alpha+3)-\sigma_{e q}{ }^{3} .
\end{aligned}
$$

The coefficients of a series are determined from statistical moments so far they were analyzed as functions of working pressure and operating time. To determine them, let make a replacement of a random variable $\hat{\sigma}_{e q}=\sigma_{e q} / \beta$ with a PDF $f\left(\hat{\sigma}_{e q}\right)$ wherein $f\left(\hat{\sigma}_{e q}\right)=f_{1}\left(\sigma_{e q} / \beta\right) / \beta$

$$
f\left(\hat{\sigma}_{e q}\right)=\sum_{n=0}^{\infty} b_{n} e^{-\hat{\sigma}_{e q}} \hat{\sigma}_{e q} \alpha_{n}^{(\alpha)}\left(\hat{\sigma}_{e q}\right)
$$

where the coefficients $b_{n}$ are expressed as follows:

$$
\begin{aligned}
b_{n}=\frac{n !}{\Gamma(n+\alpha+1)} \int_{0}^{\infty} L_{n}^{\alpha}\left(\hat{\sigma}_{e q}\right) f\left(\hat{\sigma}_{e q}\right) d \hat{\sigma}_{e q} & \\
& \frac{n !}{\Gamma(n+\alpha+1)} \int_{0}^{\infty} L_{n}^{\alpha}\left(\frac{\sigma_{e q}}{\beta}\right) f_{1}\left(\frac{\sigma_{e q}}{\beta}\right) \beta d \sigma_{e q}
\end{aligned}
$$

If we substitute the expressions of the Lager polynomials (11) in (13) and take into account the PDF normalization as well as determination of the initial statistical moments, then one can obtain:

$$
\begin{aligned}
& b_{1}=\frac{1+\alpha-m_{n 1} / \beta}{\Gamma(\alpha+2)} \\
& b_{2}=\frac{1}{\Gamma(\alpha+3)}\left[(\alpha+1)(\alpha+2)-\frac{2 m_{n 1}}{\beta}(\alpha+2)+\frac{m_{n 2}}{\beta^{2}}\right]
\end{aligned}
$$

Since in (12) and (13) the coefficients $\alpha$ and $\beta$ are arbitrary, they can be chosen (Tihonov, 1982) in such a way that $b_{1}=b_{2}=0$. Other coefficients $b_{n}$ can be expressed in terms of initial statistical moments $m_{n i}$ and will be functions of time and pressure. 


$$
\begin{gathered}
\alpha(p, t)=\frac{m_{\sigma}^{2}(p, t)}{\operatorname{Var}_{\sigma_{e q}}(p, t)}-1 ; \beta(p, t)=\frac{\operatorname{Var}_{\sigma_{e q}}(p, t)}{m_{\sigma}(p, t)}, \\
b_{0}(p, t)=\frac{1}{\Gamma(\alpha(p, t)+1)}, \quad b_{3}(p, t)=\frac{1}{\Gamma(\alpha(p, t)+4)} \\
{\left[\frac{m_{n 2}(p, t)}{\beta^{2}(p, t)}(\alpha(p, t)+3)-\frac{m_{n 3}(p, t)}{\beta^{3}(p, t)}\right]}
\end{gathered}
$$

The last is useful to express from the mean value $m_{\sigma}(p, t)$, the variance $\operatorname{Var}_{\sigma_{e q}}(p, t)$ and the asymmetry coefficient $a_{\sigma}(p, t)$.

$$
\begin{gathered}
m_{n 1}(p, t)=m_{\sigma}(p, t) \\
m_{n 2}(p, t)=m_{\sigma}^{2}(p, t)+\operatorname{Var}_{\sigma_{e q}}(p, t) \\
m_{n 3}(p, t)=a_{\sigma}(p, t) \operatorname{Var}_{\sigma_{e q}}(p, t) \sqrt{\operatorname{Var}_{\sigma_{e q}}(p, t)}+3 m_{\sigma}(p, t) \operatorname{Var}_{\sigma_{e q}}(p, t)+m_{\sigma}^{3}(p, t)
\end{gathered}
$$

After substituting the data obtained in (13) using (12), (15) - (17), the PDFs were obtained for the operating life of 35,40 and 45 years, respectively, and for different levels of workload. The indicated PDF is shown in Fig. 9. From the graphs it can be seen that with a nominal load of 3 $\mathrm{MPa}$, plastic strain may occur only during exploitation for 45 years.

After integrating the obtained PDF (12) on the internal load according to the operating time by the following formula, it can be obtained the dependence of the probability of the appearance of plasticity on the level of loading and the service time of the pipeline:

$$
Q(p, t)=1-\int_{0}^{\sigma} \frac{f_{1}\left({ }^{\sigma_{e q}} / \beta \mid p, t\right)}{\beta} d \sigma_{e q}
$$

On fig. 9 the PDF for different load level for different operating periods is shown. Vertical lines depict the attainment of yield and ultimate stress, respectively. 


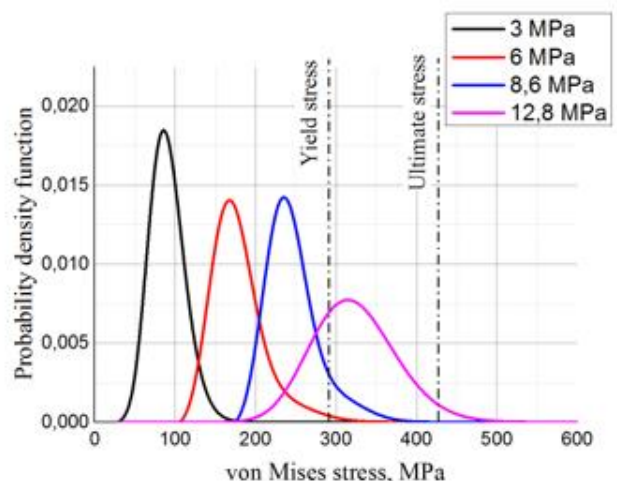

a)

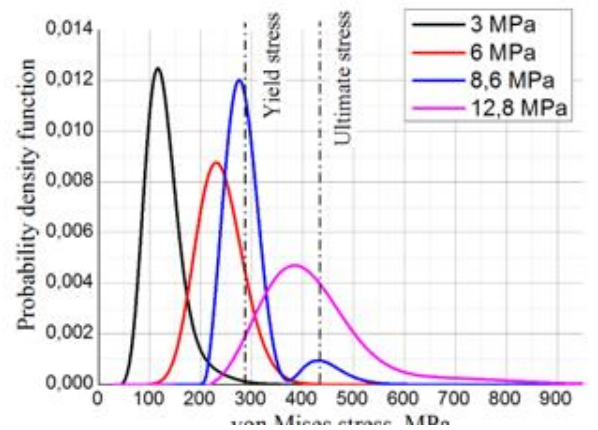

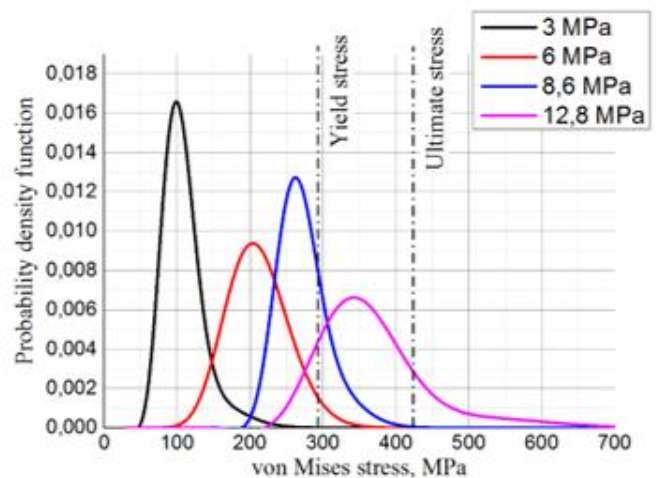

b)

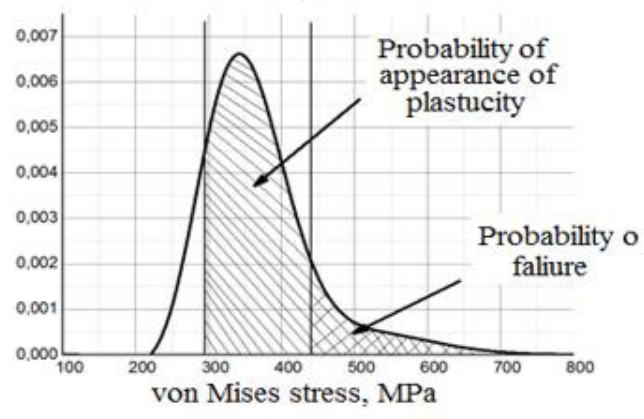

Fig. 9. The probability density functions of maximum equivalent stresses in the pipeline with damage during the period of operation of 35 (a), 40 (b) and 45 (c) years at different load levels

In Fig. 9, the area that corresponds to the probability of the appearance of plastic strains in the design (hatching with one line) is shown schematically. The area of the zone, which is hatched by two lines, is the probability that the equivalent stresses reach ultimate stress, that is, the probability of rupture.

Thus, it is possible to obtain the probability of appearance of plastic strain in the material separately for each level of loading and for the corresponding period of operation (Fig.10).

When operation for 45 years, the probability of plastic strain at a load of $12 \mathrm{MPa}$ is equal to 0.94 . From the graph (Fig. 10) it can be seen that after the pipeline operation of 40 years with the corresponding defect, the appearance of plasticity is observed not only with the maximum load in the corresponding zone, but with a typical load of $6 \mathrm{MPa}$. 


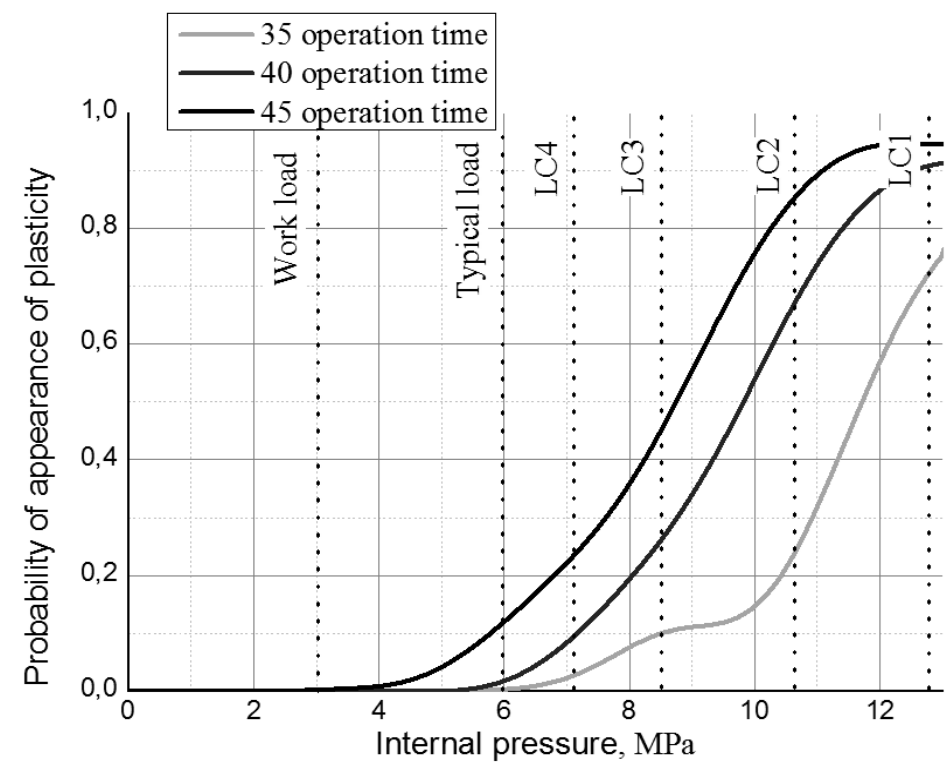

Fig. 10. The probability of appearance of plasticity in the design at different operating periods and load levels

At a maximum load of $12.8 \mathrm{MPa}$, the probability of appearance plasticity is almost equal to 1 .

\section{Conclusions}

In this paper, a series of calculations of the curvilinear part of the pipeline with defect, which develops stochastically at different operating periods, has been carried out. Based on the obtained results, the probabilistic characteristics of the stress state, such as the mean value, the coefficient of variation and asymmetry, depending on the internal pressure and the time has been determined. On the basis of these data, PDF were approximated for different designs operation time at appropriate loading levels. It has been determined that during operation of the pipeline elbow with defect, which has been developed during the exploitation period from 35 to 45 years, there is a probability appearing of plastic strain, correspondingly from 0,117 to 0,94 at the maximum possible load, and with a typical load of $6 \mathrm{MPa}$ at the operation time from 45 years the probability of plasticity increases and equal to 0,125 . It means, that during operation time more than 40 years, pipeline with corresponding defect and load level can be broken.

Outlook It should also be mentioned that in the structure significant plastic strains appear and accumulation of fatigue damage may occur, which can have the reduction of the residual life of the pipeline element and requires a separate analysis.

\section{References}

Ahammed M (1997). Prediction of remaining strength of corroded pressurised pipelines. Int. J. Pres. Ves. Ki Pq q, 161(96), 213-217. https://doi.org/10.1016/S0308-0161(96)00081-6 Bazán FAV and Beck AT (2013). Stochastic process corrosion growth models for pipeline 
reliability. Corrosion Science, 74, 50-58. https://doi.org/10.1016/j.corsci.2013.04.011

Caleyo F, Velázquez JC, Valor A, \& Hallen JM (2009). Probability distribution of pitting corrosion depth and rate in underground pipelines: A Monte Carlo study. Corrosion Science, 51(9), 1925-1934. https://doi.org/10.1016/j.corsci.2009.05.019

Chen Y, Zhang H, Zhang J, Liu X, Li X, \& Zhou J (2015). Failure assessment of X80 pipeline with interacting corrosion defects. Engineering Failure Analysis, 47(PA), 67-76. https://doi.org/10.1016/j.engfailanal.2014.09.013

Cunha DJS, Benjamin AC, Silva RCC, Guerreiro JNC, \& Drach PRC (2014). Fatigue analysis of corroded pipelines subjected to pressure and temperature loadings. International Journal of Pressure Vessels and Piping, 113, 15-24. https://doi.org/10.1016/j.ijpvp.2013.10.013

da Costa-Mattos, H S, Reis JML, Sampaio RF, \& Perrut VA (2009). An alternative methodology to repair localized corrosion damage in metallic pipelines with epoxy resins. Materials and Design, 30(9), 3581-3591. https://doi.org/10.1016/j.matdes.2009.02.026

De Leon D, \& Macías OF (2005). Effect of spatial correlation on the failure probability of pipelines under corrosion. International Journal of Pressure Vessels and Piping, 82(2), 123-128. https://doi.org/10.1016/j.ijpvp.2004.07.018

Khalaj Khalajestani M \& Bahaari MR (2014). Investigation of pressurized elbows containing interacting corrosion defects. International Journal of Pressure Vessels and Piping, 123, 77-85. https://doi.org/10.1016/j.ijpvp.2014.08.002

Larin O, Barkanov E, \& Vodka O (2016). Prediction of reliability of the corroded pipeline considering the randomness of corrosion damage and its stochastic growth. Engineering Failure Analysis, 66, 60-71. https://doi.org/10.1016/j.engfailanal.2016.03.022

Larin O, \& Vodka O (2015). A probability approach to the estimation of the process of accumulation of the high-cycle fatigue damage considering the natural aging of a material. International Journal of Damage Mechanics, 24(2), 294-310. https://doi.org/10.1177/1056789514536067

Li S X, Yu SR, Zeng H L, Li JH, \& Liang R (2009). Predicting corrosion remaining life of underground pipelines with a mechanically-based probabilistic model. Journal of Petroleum Science and Engineering, 65(3-4), 162-166. https://doi.org/10.1016/j.petrol.2008.12.023

Ma B, Shuai J, Liu D, \& Xu K (2013). Assessment on failure pressure of high strength pipeline with corrosion defects. Engineering Failure Analysis, 32, 209-219. https://doi.org/10.1016/j.engfailanal.2013.03.015

Mirchev Y, Larin OO, \& Potopalska KE (2016). Investigation of influence of the repair bandage on the stress-strain state of the pipeline elbow with VSD. „NDT Days 2016”/ „Дии На Безразрушителния Контрол 2016”, (1(187)), 411-414.

Netto T A, Ferraz US, \& Botto A (2007). On the effect of corrosion defects on the collapse pressure of pipelines. International Journal of Solids and Structures, 44(22-23), 75977614. https://doi.org/10.1016/j.ijsolstr.2007.04.028

Silva, RCC, Guerreiro, JNC \& Loula, AFD (2007). A study of pipe interacting corrosion defects using the FEM and neural networks. Advances in Engineering Software, 38(11-12), 868875. https://doi.org/10.1016/j.advengsoft.2006.08.047

Teixeira AP, Guedes Soares C, Netto T A, \& Estefen SF (2008). Reliability of pipelines with corrosion defects. International Journal of Pressure Vessels and Piping, 85(4), 228-237. https://doi.org/10.1016/j.ijpvp.2007.09.002

Tihonov VI (1982). Statisticheskaya radiotehnika. Moscow: "RADIO I SVYAZ."

Valor A, Caleyo F, Alfonso L, Rivas D, \& Hallen JM (2007). Stochastic modeling of pitting corrosion: A new model for initiation and growth of multiple corrosion pits. Corrosion Science, 49(2), 559-579. https://doi.org/10.1016/j.corsci.2006.05.049

Valor A, Caleyo F, Hallen JM, \& Velázquez JC (2013). Reliability assessment of buried pipelines based on different corrosion rate models. Corrosion Science, 66, 78-87. 
https://doi.org/10.1016/j.corsci.2012.09.005

Vodka O (2015). Computation tool for assessing the probability characteristics of the stress state of the pipeline part defected by pitting corrosion. Advances in Engineering Software, 90, 159-168. https://doi.org/10.1016/j.advengsoft.2015.08.012 